\title{
Treatment Patterns with Disease-Modifying Antirheumatic Drugs in U.S. Veterans with Newly Diagnosed Rheumatoid Arthritis, Psoriatic Arthritis, or Ankylosing Spondylitis
}

\author{
Jessica A. Walsh, MD; Shaobo Pei, PhD; Gopi K. Penmetsa, MD; Brian C. Sauer, PhD; Vikas Patil, MS; \\ Jodi H. Walker, PharmD, BCPS; Jerry Clewell, PharmD, MBA; Kevin M. Douglas, MD; \\ Daniel O. Clegg, MD; Grant W. Cannon, MD; and Ahmad Halwani, MD
}

\section{ABSTRACT}

BACKGROUND: Delays in treatment for inflammatory arthritis (IA) are associated with unfavorable outcomes, including impaired quality of life, irreversible joint damage, and disability.

OBJECTIVE: To characterize treatment initiation patterns in veterans with newly diagnosed rheumatoid arthritis (RA), psoriatic arthritis (PsA), or ankylosing spondylitis (AS).

METHODS: ICD-9/10-CM codes and natural language processing were used to identify incident cases of RA, PsA, or AS between January 1 , 2007, and December 31, 2015, in patients enrolled in the Veterans Health Administration. Patterns of treatment initiation and nontreatment with disease-modifying antirheumatic drugs (DMARDs) were assessed in the 12-month follow-up period after the incident diagnosis. Outcomes included the percentage of veterans treated with a DMARD, the mean time to the initial DMARD after diagnosis, and the percentage of veterans who accessed rheumatology care before DMARD initiation. To assess outcomes over time, veterans were grouped by year of initial IA diagnosis. Additionally, outcomes were compared between nonbiologic and biologic DMARDs and among IA subtypes (RA, PsA, and AS). Groups were statistically compared with $95 \%$ confidence intervals.

RESULTS: The population consisted of 12,118 IA veterans (9,711 RA, 1,472 PSA, and $935 \mathrm{AS}$ ), with $91.3 \%$ males and a mean age of 63.7 years. The percentage of veterans treated with $\geq 1$ DMARD (nonbiologic or biologic) during the 12-month follow-up period increased from $48.8 \%$ in 2007 to $66.4 \%$ in 2015 . In veterans diagnosed with IA in 2015, DMARD treatment was more common for PsA patients (72.9\%) and RA patients (68.6\%) than for AS patients (28.9\%). In the subset treated with a DMARD within 12 months after diagnosis, the mean time to the initial DMARD after diagnosis did not change throughout the observation period ( 35.5 days for RA, 43.9 days for PSA, and 59.5 days for AS). Rheumatology specialty care was accessed by $87.4 \%$ of veterans treated with a nonbiologic DMARD and $92.2 \%$ of veterans treated with a biologic DMARD, in patients diagnosed in 2015.

CONCLUSIONS: DMARD treatment rates during the initial 12 months after diagnosis increased between 2007 and 2015, but nontreatment remained common, particularly in veterans with AS. The time to treatment after diagnosis was stable over time; it was shortest for RA, intermediate for PsA, and longest for AS. DMARD treatment was uncommon in veterans who did not access rheumatology specialty care.

J Manag Care Spec Pharm. 2019;25(11):1218-26

Copyright $\odot 2019$, Academy of Managed Care Pharmacy. All rights reserved.

\section{What is already known about this subject}

Treatment delays and nontreatment for inflammatory arthritis (IA) have been reported in various populations and contribute to quality-of-life limitations, functional impairment, and irreversible joint damage.

Treatment guidelines from the American College of Rheumatology and the European League Against Rheumatism recommend that treatment with disease-modifying antirheumatic drugs (DMARDs) should begin as soon as the diagnosis of rheumatoid arthritis is made, and multiple studies have demonstrated the benefits of early DMARD treatment for rheumatoid arthritis (RA), psoriatic arthritis (PsA), and ankylosing spondylitis (AS).

Estimates of treatment delays and nontreatment within the Veterans Affairs Health Care System patients are important for understanding potential opportunities to improve care for veterans with IA.

\section{What this study adds}

DMARD nontreatment during the 12 months after the initial diagnosis of IA was common in U.S. veterans, but rates of nontreatment improved over time (51\% in 2007 to 34\% in 2015).

Nontreatment rates were higher for AS than RA and PsA (71.1\%,

$31.4 \%, 27.1 \%$, respectively, in 2015), and the mean time to treatment after diagnosis was longer for AS than RA and PsA (59.5, 35.5, and 43.9 days, respectively).

DMARD treatment was uncommon in veterans who did not access rheumatology specialty care (12.6\% with nonbiologics, 7.9\% with biologics in 2015).

T nflammatory arthritis (IA) is a group of autoimmune inflammatory diseases characterized by chronic pain, systemic inflammation, and damage to joints and other tissues. Common forms of IA include rheumatoid arthritis (RA), psoriatic arthritis (PsA), and ankylosing spondylitis (AS). $\mathrm{RA}$ is characterized by symmetric and erosive polyarticular peripheral arthritis. PsA is a heterogeneous disease that may present as oligoarticular or polyarticular arthritis and may affect the entheses, tendons, and axial skeleton. AS, the prototype of axial spondyloarthritis (SpA), is characterized by 


\section{FIGURE 1 Patient Selection Protocol}

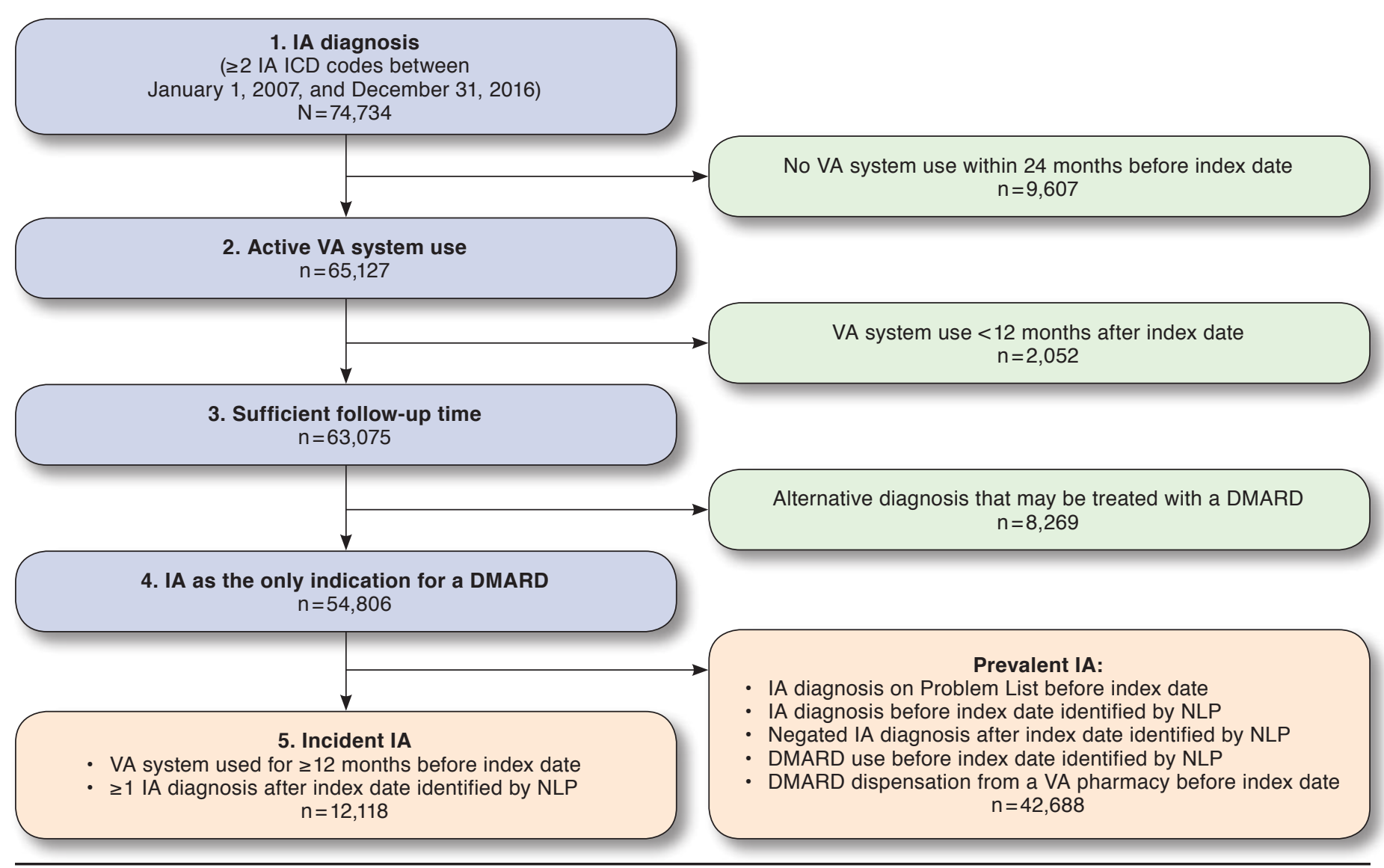

DMARD = disease-modifying antirheumatic drug; IA = inflammatory arthritis; ICD = International Classification of Diseases; NLP=natural language processing; VA = Veterans Affairs.

inflammation in the sacroiliac joints and spine and may involve inflammatory manifestations in the peripheral joints, entheses, and eyes. The estimated prevalence of IA is 1\%-3\%, affecting an estimated 3.2 to 10 million individuals in the United States. ${ }^{1}$

IA produces substantial burdens for individuals and society. In a rheumatic disease population (51.4\% RA, 23.8\% axial SpA, and $19.6 \%$ PsA), patients reported that their disease had moderate to severe effects on their quality of life (51.9\%), work/ daily activities (49.2\%), emotional well-being (41.0\%), personal relationships (26.0\%), and close relatives' lives (32.3\%). ${ }^{2}$ In an RA study, 39\% of employees with early disease suffered from work disability after 10 years, ${ }^{3}$ and $50 \%$ reported disability in an SpA population composed of 39\% AS patients and 36\% PsA patients. ${ }^{4}$ In terms of cost, the annual direct costs in an RA population were approximately 10.6 billion, and indirect costs were 36.7 billion, in 2013 U.S. dollars. ${ }^{3}$ In AS patients, compared with matched controls, the median direct health care costs were 10 times higher $(\$ 24,978$ vs. $\$ 2,139$ per patient per year in 2015 U.S. dollars)..$^{5}$ Thus, IA broadly affects patients, family members, employers, health care payers, and society.

In the past 2 decades, effective options for IA treatments have greatly expanded. Treatment with disease-modifying antirheumatic drugs (DMARDs), particularly in early stages of disease, improves outcomes such as quality of life, physical function, clinical treatment responses, radiographic joint damage, and disability. ${ }^{6-17}$ According to expert consensus, patients with active IA should be treated with a DMARD (nonbiologic or biologic), barring a contra-indication or patient refusal. Specifically, treatment guidelines from the American College of Rheumatology (ACR) and European League Against Rheumatism (EULAR) recommend that patients with active PsA or RA should be treated with a DMARD as first-line therapy, ${ }^{18,19}$ and DMARDs (biologic) are recommended for AS patients with intolerance or inadequate response to a 4-week trial of nonsteroidal anti-inflammatory drugs (NSAIDs). ${ }^{20}$ Furthermore, guidelines recommend that DMARD treatment 
should begin as soon as the IA diagnosis is made. ${ }^{21}$ Since DMARD treatment is a top priority for IA patients, DMARD use became the first rheumatology-specific quality measure for assessing health systems with the Health Plan Employer Data and Information Set (HEDIS). ${ }^{22}$

Studies in U.S. administrative claims datasets have raised concerns regarding the underuse of DMARDs. For example, in Medicare and commercially insured populations, rates of DMARD treatment ranged from 30\%-63\% in RA patients. ${ }^{23}$ The goal of this study was to characterize treatment patterns in Veterans Affairs (VA) patients with newly diagnosed IA. We quantified rates of DMARD nontreatment and initiation delays within the 12 months after the initial IA diagnosis and characterized trends in DMARD treatment over time in all incident IA cases and in subgroups with RA, PsA, and AS.

\section{Methods}

\section{Data Sources}

This study used historical data from veterans enrolled in the Veterans Health Administration (VHA). The data source was the Corporate Data Warehouse, a national repository of data from the VHA medical record system (VistA) and other VHA clinical and administrative systems. ${ }^{24}$ The patient Integration Control Number was used to link patients across VHA stations. Data were housed and analyzed within the Veterans Affairs Informatics and Computing Infrastructure. ${ }^{25}$ This research was conducted in compliance with the Helsinki Declaration, with the approval of the University of Utah Institutional Review Board.

\section{Population}

Cohort Selection. The cohort was assembled in 5 steps (Figure 1). First, patients were identified with $\geq 2$ International Classification of Diseases, Ninth/Tenth Revision, Clinical Modification (ICD-9/10-CM) billing codes on separate days for RA (714.x, M05.x, M06.x), PsA (696.0, L40.5x), or AS (720.0, M45.x), during the observation period (January 1, 2007-December 31, 2016; Appendix A, available in online article). The earliest ICD code date (index date) was required to be within the enrollment period (January 1, 2007December 31, 2015).

Second, veterans who were actively using the VA system before the index date were selected (defined by an encounter with a provider or a medication dispensation $\leq 24$ months before the initial IA diagnosis code).

Third, patients with sufficient follow-up time were selected (VA system use for $\geq 12$ months after the index date).

Fourth, patients were selected if IA was their only diagnosis for which DMARD treatment would be appropriate. Patients were excluded if they had an ICD code for an alternative diagnosis that may be treated with a DMARD before the index date or during the 12-month follow-up period. Excluded alternative diagnoses were chronic lymphocytic leukemia, connective tissue disease, Crohn disease, cutaneous lupus, malaria, nonHodgkin lymphoma, noninfectious uveitis, organ transplant, Paget disease, polymyalgia rheumatic, porphyria cutanea tarda, sarcoidosis, ulcerative colitis, and vasculitis

Fifth, patients with an incident IA diagnosis were selected. The inclusion criteria for incident IA were (a) VA system use for $\geq 12$ months before the index date and (b) $\geq 1$ IA diagnosis identified by natural language processing (NLP) in clinical notes after the index date. Patients were excluded if there was evidence of an IA diagnosis before the index date (prevalent IA). Criteria for prevalent IA were (a) IA diagnosis on the problem list before the index date, (b) IA diagnosis in a clinic note identified by NLP before the index date, (c) a negated IA diagnosis identified by NLP after the index date, (d) DMARD use identified by NLP before the index date, and (e) DMARD dispensation from the pharmacy before the index date.

The NLP used for identifying incident IA cases applied a rule-based approach for affirming or negating specific terms relating to IA diagnostic and treatment language. ${ }^{26,27}$ The IA diagnostic terms for NLP included rheumatoid, psoriatic arthritis, ank* spond*, spondyloarthritis, spondyloarthritidies, spondyloarthropathy, spondyloarthropathies, psoriasis with arthritis, inflam* arth", and RA. RA terms representing "right atrium" and "room air" were excluded. A previously validated implementation of the ConText algorithm in $\mathrm{R}$ version 3.4.0 ( $\mathrm{R}$ Foundation for Statistical Computing, Vienna Austria) was used to detect the presence of diagnostic terms in clinical care notes. ${ }^{27-29}$

Cohort Validation. Chart review was performed by 2 rheumatologists (coauthors JAW and GKP) to establish criteria for patient selection. The chart review population consisted of a training subset $(\mathrm{n}=118)$ for developing incident IA criteria and an independent testing subset $(\mathrm{n}=151)$ for quantifying the accuracy of the criteria. The a priori accuracy goal was $\geq 80 \%$. For each patient, the chart reviewers confirmed or negated the IA diagnosis and estimated the initial date of diagnosis. Patients with evidence of an IA diagnosis per chart review before the index date were classified as prevalent cases. Patients without a confirmed diagnosis of IA were classified as non-IA cases. The remainder of cases (with a confirmed IA diagnosis and no evidence of an IA diagnosis before the index date) were classified as incident IA cases. The population was subdivided into groups according to the calendar year of the initial IA diagnosis.

\section{Variables}

Outcome variables included (a) percentage of veterans with DMARD treatment during the 12-month follow-up period, (b) mean number of days to the initial DMARD after IA diagnosis 
Treatment Patterns with Disease-Modifying Antirheumatic Drugs in U.S. Veterans with

Newly Diagnosed Rheumatoid Arthritis, Psoriatic Arthritis, or Ankylosing Spondylitis

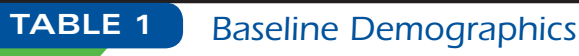

\begin{tabular}{|c|c|c|c|c|c|c|c|c|c|c|c|c|}
\hline \multirow{3}{*}{ Age } & \multicolumn{3}{|c|}{ All IA $(n=12,118)$} & \multicolumn{3}{|c|}{$\mathrm{RA}(\mathrm{n}=9,711)$} & \multicolumn{3}{|c|}{ PsA $(n=1,472)$} & \multicolumn{3}{|c|}{ AS $(n=935)$} \\
\hline & \multicolumn{3}{|c|}{ N/Mean (\%/SD) [95\% CI] } & \multicolumn{3}{|c|}{ N/Mean (\%/SD) [95\% CI] } & \multicolumn{3}{|c|}{ N/Mean (\%/SD) $[95 \% \mathrm{CI}]$} & \multicolumn{3}{|c|}{ N/Mean (\%/SD) [95\% CI] } \\
\hline & 63.7 & $(13.3)$ & [63.4-63.9] & 65.2 & $(12.6)$ & {$[64.9-65.4]$} & 57.8 & $(13.1)$ & [57.1-58.5] & 57.6 & $(16.1)$ & {$[56.5-58.6]$} \\
\hline Male & 11,060 & (91.3) & [90.8-91.8] & 8,798 & $(90.6)$ & [90.0-91.2] & 1,376 & $(93.5)$ & [92.1-94.6] & 886 & $(94.8)$ & [93.1-96.0] \\
\hline \multicolumn{13}{|l|}{ Ethnicity } \\
\hline Hispanic & 618 & $(5.1)$ & {$[4.7-5.5]$} & 487 & $(5.0)$ & {$[4.6-5.5]$} & 93 & $(6.3)$ & {$[5.2-7.7]$} & 38 & $(4.1)$ & {$[3.0-5.5]$} \\
\hline Non-Hispanic & 10,932 & $(90.2)$ & {$[89.7-90.7]$} & 8,746 & $(90.1)$ & {$[89.5-90.6]$} & 1,320 & $(89.7)$ & {$[88.0-91.1]$} & 866 & $(92.6)$ & [90.8-94.1] \\
\hline Unknown & 568 & $(4.7)$ & {$[4.3-5.1]$} & 478 & $(4.9)$ & {$[4.5-5.4]$} & 59 & $(4.0)$ & {$[3.1-5.1]$} & 31 & (3.3) & {$[2.4-4.7]$} \\
\hline \multicolumn{13}{|l|}{ Race } \\
\hline White & 9,281 & $(76.6)$ & {$[75.8-77.3]$} & 7,302 & $(75.2)$ & {$[74.3-76.0]$} & 1,247 & $(84.7)$ & {$[82.8-86.5]$} & 732 & $(78.30$ & {$[75.5-80.8]$} \\
\hline Black & 1,600 & $(13.2)$ & [12.6-13.8] & 1,407 & $(14.5)$ & [13.8-15.2] & 90 & $(6.1)$ & {$[5.0-7.5]$} & 103 & $(11.0)$ & {$[9.2-13.2]$} \\
\hline Other & 642 & $(5.3)$ & {$[4.9-5.7]$} & 508 & $(5.2)$ & {$[4.8-5.7]$} & 75 & $(5.1)$ & [4.1-6.3] & 59 & $(6.3)$ & [4.9-8.1] \\
\hline Unknown & 595 & $(4.9)$ & {$[4.5-5.3]$} & 494 & $(5.1)$ & {$[4.7-5.5]$} & 60 & $(4.1)$ & {$[3.2-5.2]$} & 41 & $(4.4)$ & [3.3-5.9] \\
\hline \multicolumn{13}{|c|}{ Geographic region at cohort entry } \\
\hline Southeast & 2,119 & $(17.5)$ & [16.8-18.2] & 1,701 & $(17.5)$ & [16.8-18.3] & 222 & $(15.1)$ & [13.3-17.0] & 196 & $(20.7)$ & [18.5-23.7] \\
\hline North Atlantic & 3,129 & (25.8) & {$[25.1-26.6]$} & 2,546 & $(26.2)$ & {$[25.4-27.1]$} & 406 & (27.6) & [25.4-29.9] & 177 & (18.9) & [16.6-21.6] \\
\hline Midwest & 2,862 & $(23.6)$ & {$[22.9-24.4]$} & 2,336 & $(24.1)$ & [23.2-24.9] & 325 & $(22.1)$ & [20.0-24.3] & 201 & $(21.5)$ & [19.0-24.2] \\
\hline Continental & 2,123 & (17.5) & [16.9-18.2] & 1,691 & (17.4) & [16.7-18.3] & 260 & (17.7) & [15.8-19.7] & 172 & (18.4) & [16.0-21.0] \\
\hline Pacific & 1,885 & (15.6) & [14.9-16.2] & 1,437 & (14.8) & [14.1-15.5] & 259 & (17.6) & [15.7-19.6] & 189 & $(20.2)$ & [17.8-22.9] \\
\hline
\end{tabular}

in veterans treated with a DMARD during the 12-month follow-up period, and (c) rheumatology subspecialty care utilization within 12 months before DMARD dispensation. Outcomes were evaluated in the entire population (All IA) and in subsets with RA, PsA, and AS.

Patients with nonbiologic and a biologic DMARDs dispensed on the same day, as the initial DMARDs after the index date, were included in the nonbiologic and biologic DMARD categories. DMARD treatment was defined as a dispensation of the DMARD from a VA pharmacy or documentation by a VA provider of a DMARD from an outside pharmacy in the non-VA medication database. Assessed nonbiologic DMARDs included apremilast, azathioprine, cyclosporine, hydroxychloroquine, leflunomide, methotrexate, sulfasalazine, and tofacitinib. Biologic DMARDs included abatacept, adalimumab, anakinra, certolizumab, etanercept, golimumab, infliximab, rituximab, tocilizumab, and ustekinumab.

The provider specialty was assigned as rheumatology if the patient had $\geq 1$ VA rheumatology visit within 365 days before DMARD dispensation from a VA pharmacy. Provider specialty was assigned as nonrheumatology if there was no VA rheumatology visit during the study period before DMARD dispensation. Provider specialty was categorized as unknown for veterans with a DMARD recorded by a VA provider as a "non-VA medication" (i.e., dispensed by a non-VA pharmacy).

\section{Results}

\section{Cohort}

The population consisted of 12,118 veterans with IA, including 9,711 (80.1\%) with RA, 1,472 (12.1\%) with PsA, and 935
(7.7\%) with AS (Table 1). The mean age for the total cohort was 63.7 years, and $91.3 \%$ were male. White race was reported by $76.6 \%$ of patients, and $90.2 \%$ reported non-Hispanic ethnicity. The mean age was higher for RA (65.2) than PsA (57.8) and AS (57.6), and RA patients were less frequently male (90.6\%) than PsA (93.5\%) and AS (94.8\%) patients.

The accuracy of the patient selection protocol for identifying patients with incident IA was $83.3 \%$ (95\% confidence interval $[\mathrm{CI}]=69.4-91.7)$ in the training set. In the independent testing set, the accuracy of the patient selection protocol was $80.8 \%$ (95\% CI $=73.8-86.3)$. Among the $19.3 \%$ (95\% CI $=13.7$ 26.2) of misclassified patients in the testing set, $6.0 \%$ (95\% $\mathrm{CI}=3.2-10.9)$ did not have an IA diagnosis, and 13.3\% (95\% $\mathrm{CI}=8.7-19.6)$ were prevalent IA cases.

\section{Treatment Within 12 Months of Diagnosis}

DMARD treatment (nonbiologic or biologic) within the 12-month follow-up period after diagnosis increased between 2007 and 2015 (48.8\% [95\% CI=46.3-51.4] in 2007 to $66.4 \%$ [95\% CI=63.7-68.9] in 2015; Figure 2). Treatment rates increased over time with the nonbiologics and biologics subgroups (nonbiologics: 46.1\% [95\% CI =43.5-48.7] in 2007 to $61.4 \%[95 \% \mathrm{CI}=58.7-64.1]$ in 2015 ; biologics: $8.8 \%[95 \%$ $C I=7.4-10.4]$ in 2007 to $15.4 \%$ [95\% CI $=13.5-17.5]$ in 2015). DMARD treatment (biologic or nonbiologic) was less frequent for AS than RA and PsA throughout the observation period (AS: 28.9\% [95\% CI=20.5-39.0]; RA: 68.6\% [95\% CI $=65.6$ 71.4]; and PsA: 72.9\% [95\% CI=66.1-78.7] in 2015; Figure 3).

The percentage of veterans with DMARD treatment statistically increased in the RA subgroup between 2007 and 2015 


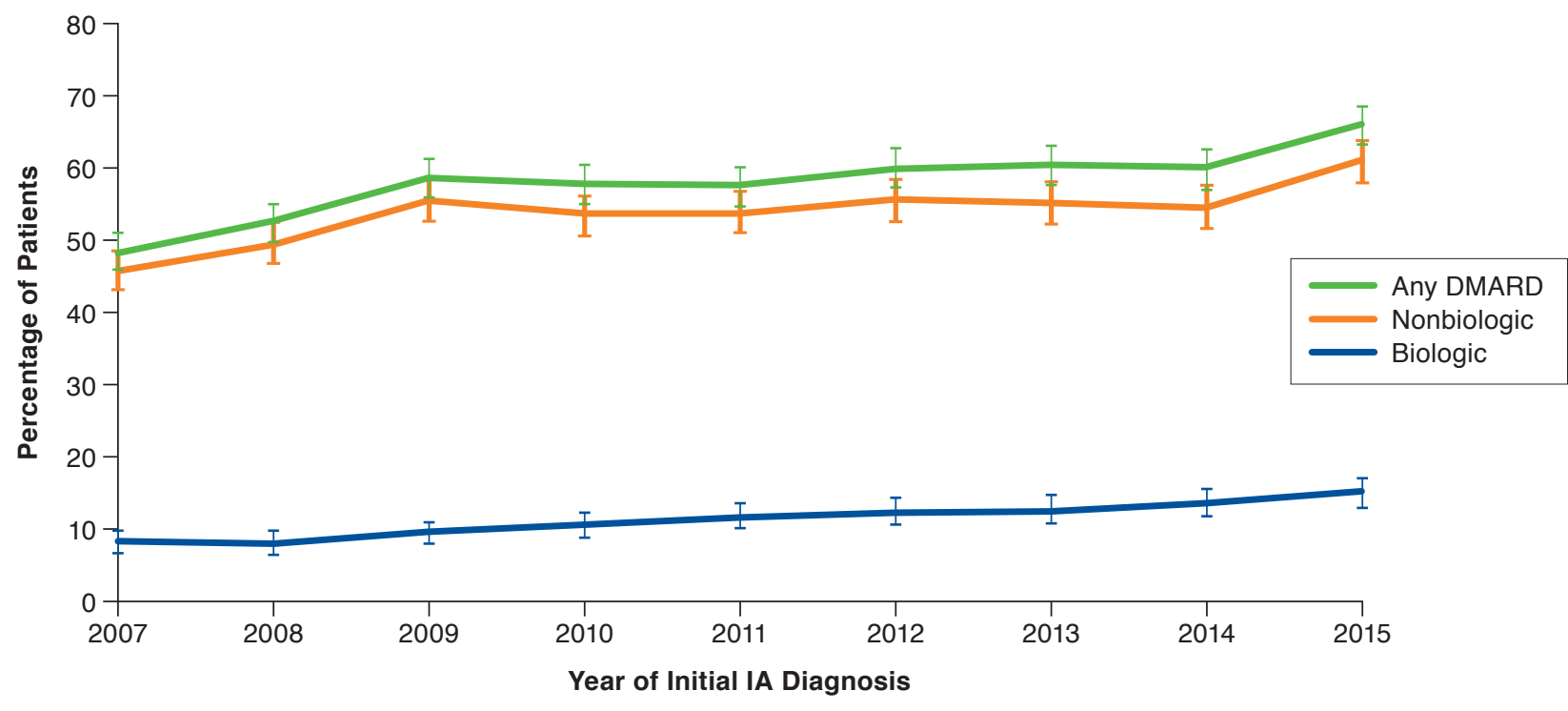

DMARD = disease-modifying antirheumatic drug; IA = inflammatory arthritis.

(50.4\% [95\% CI $=47.5-53.2]$ to $68.6 \%[95 \% \mathrm{CI}=65.6-71.4])$. The percentage of veterans with PsA receiving a DMARD numerically increased between 2007 and 2015, but the difference was not statistically significant $(58.7 \%$ [95\% CI $=50.8$ 66.2] vs. $72.9 \%$ [95\% CI=66.1-78.7]). Similarly, the percentage with AS who received a DMARD increased numerically but not statistically between 2007 and 2015 (20.2\% [95\% CI =13.8$28.5]$ vs. $28.9 \%$ [95\% CI=20.5-39.0]).

\section{Time Between Diagnosis and Treatment}

In veterans with DMARD treatment during the 12-month follow-up period, the mean number of days between the initial IA diagnosis and the initial DMARD did not change between 2007 and 2015 (36.9 [95\% CI=35.2-38.6] days for nonbiologic DMARDs or 122.0 [95\% CI=116.4-127.6] days for biologic DMARDs, averaged over the observation period; Figure 4). Comparisons between IA subtypes demonstrated that the mean number of days between diagnosis and initial DMARD (nonbiologic or biologic) was shortest for RA, intermediate for PsA, and longest for AS (RA: 35.5 days [95\% CI=33.8-37.2]; PsA: 43.9 days [95\% CI=39.2-48.6]; and AS: 59.5 days [95\% CI $=49.9-69.0]$, averaged across time).

\section{Rheumatology Care}

Throughout the observation period, rheumatology specialty care within the VA system was accessed before DMARD initiation by $78.0 \%-92.2 \%$ of veterans treated with a DMARD (non- biologic or biologic; Appendix B, available in online article). The percentage of patients who accessed rheumatology care statistically increased throughout the observation period for the group with a nonbiologic as their initial DMARD (79.0\% [95\% CI $=75.4-82.2]$ in 2007 to $87.4 \%$ [95\% CI $=84.6-89.7$ ] in 2015). The percentage of veterans who accessed rheumatology care was statistically similar in all years for the group treated with a biologic as their initial DMARD (range: $83.7 \%$ [95\% CI $=75.4-89.5]$ to $92.2 \%[95 \% \mathrm{CI}=87.3-95.3]$ ). Averaged across study years, the percentage of veterans who accessed rheumatology care before DMARD treatment (nonbiologic or biologic) was lowest for RA and highest for AS (RA: 83.2\% [95\% CI =82.2-84.2]; PsA: 86.1\% [95\% CI =83.9-88.1]; and AS: $88.5 \%[95 \% \mathrm{CI}=84.3-91.8]$ ).

\section{Discussion}

Approximately one third to one half of veterans with IA were not treated with a DMARD (nonbiologic or biologic) within 12 months after their initial IA diagnosis. Similar rates of DMARD nontreatment were reported for commercially insured and Medicare beneficiaries with incident RA, ranging from 28\% to $45 \%$ during follow-up periods, ranging from 1 year to a mean of 3.5 years after diagnosis. ${ }^{30-34}$ These high rates of nontreatment raise concern for lost opportunities to improve outcomes. In RA, prompt treatment after disease onset is well established as a major predictor of favorable long-term clinical, functional, and radiographic outcomes. ${ }^{10}$ Similarly, treatment in early 


\section{FIGURE 3 Percentage of Veterans with RA, PsA, and AS Treated with $\geq 1$ DMARD (Nonbiologic or Biologic)} Within 12 Months After Diagnosis

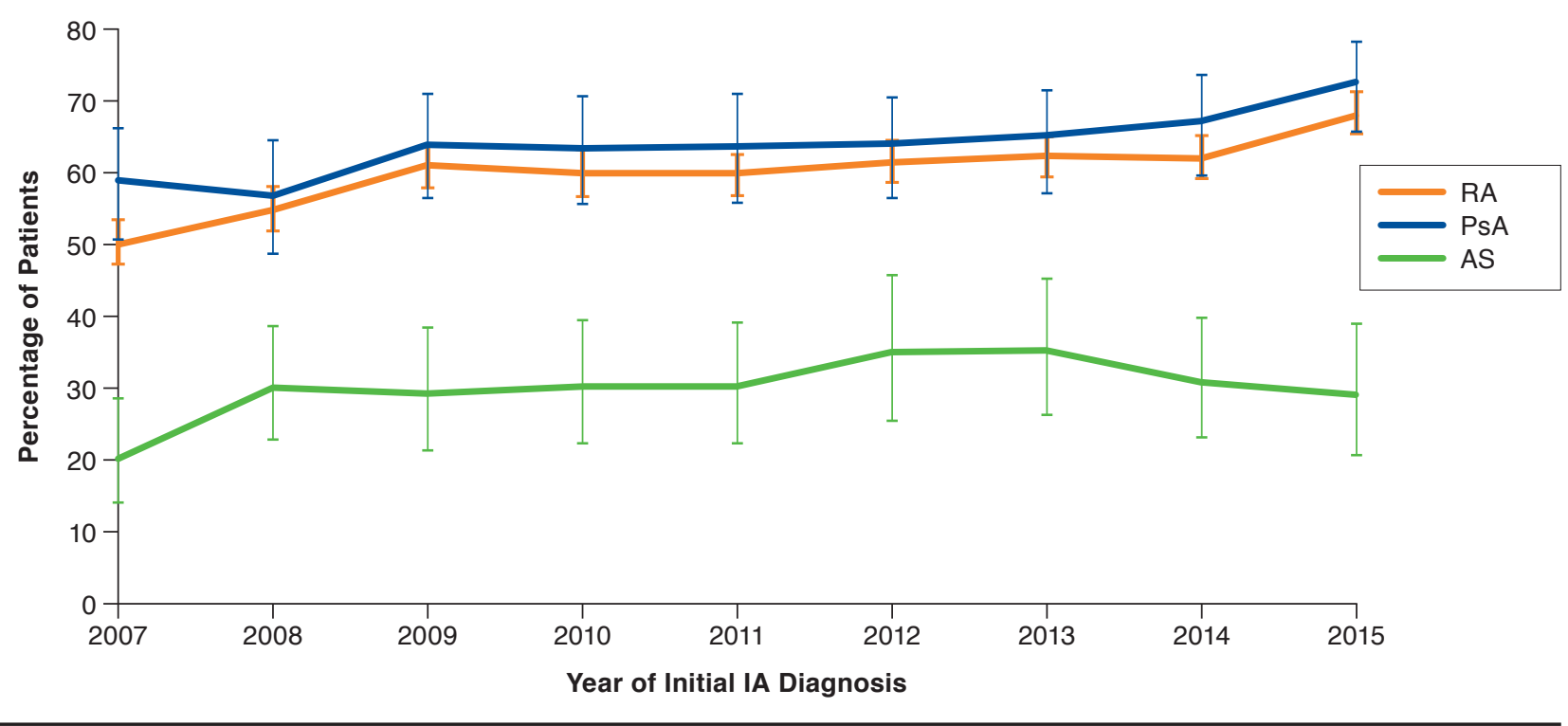

AS = ankylosing spondylitis; DMARD = disease-modifying antirheumatic drug; IA = inflammatory arthritis; PsA=psoriatic arthritis; $R A=$ rheumatoid arthritis.

stages of AS and PsA improves long-term clinical and radiographic outcomes. ${ }^{35-38}$ Thus, understanding and minimizing treatment delays is critical to optimizing care for IA patients.

Among veterans treated with a DMARD (nonbiologic or biologic) during the 12-month follow-up period, the mean time between initial diagnosis and treatment was 37.5 days. This time interval was similar to the 39-day interval reported in a claims database study of patients with commercial and Medicare insurance (MarketScan). ${ }^{33}$ In contrast, time to initial DMARD in a U.S. military TRICARE claims database was 125.4 days after diagnosis. ${ }^{8}$ Differences in study design and populations likely contributed to differences in the treatment delays. For example, the follow-up period in the TRICARE study was 2 years, compared with 1 year in the VA and the MarketScan studies. Additionally, the TRICARE population was younger than the VA and MarketScan populations (mean ages 47.2, 63.7, and 58.6 years, respectively). Lower health care utilization was associated with longer treatment delays in the TRICARE population, and it is likely that health care utilization was lower in the younger and presumably healthier TRICARE patients, compared with the older VA and MarketScan populations.

In this veteran population, there were differences in treatment patterns between IA subtypes. In particular, AS patients were less commonly treated with DMARDs than RA and PsA patients (29\% AS, 69\% RA, and 73\% PsA in 2015). AS differs from RA and PsA in that DMARDs (biologic) are recommended as second-line treatment for AS, rather than first-line treatment. ${ }^{18-20}$ However, the recommended first-line treatment, with NSAIDs, is often inadequate or contra-indicated, ${ }^{39}$ and the recommended NSAID trial period, before DMARD initiation, is only 4 weeks. Thus, the $71 \%$ nontreatment rate for AS during the 12 months after diagnosis suggests that many AS patients were undertreated with DMARDs.

The more frequent use of biologics than nonbiologics in AS was expected, since nonbiologics are ineffective for the axial manifestations that are the hallmark of AS. ${ }^{20}$ In contrast, nonbiolologics and biologics are effective for RA, and nonbiologics are typically required by payers as first-line treatment because of lower cost. Historically, in PsA, the first-line treatment was a nonbiologic DMARD, but patterns are changing, with growing evidence that biologics, but not nonbiologics, improve clinical and radiographic outcomes in PsA. ${ }^{19,40-43}$ These changes likely contributed to the slightly higher rates of biologic DMARDs in PsA than RA as the initial DMARD after diagnosis.

DMARD treatment (biologic and nonbiologic) was infrequent in newly diagnosed IA patients who did not access rheumatology subspecialty care before DMARD dispensation (13\% with nonbiologics and 8.0\% with biologics in 2015). Similarly, in other studies, IA patients who received care from a rheumatology provider were 2-7 times more likely to receive DMARD treatment, compared with patients who were not receiving 


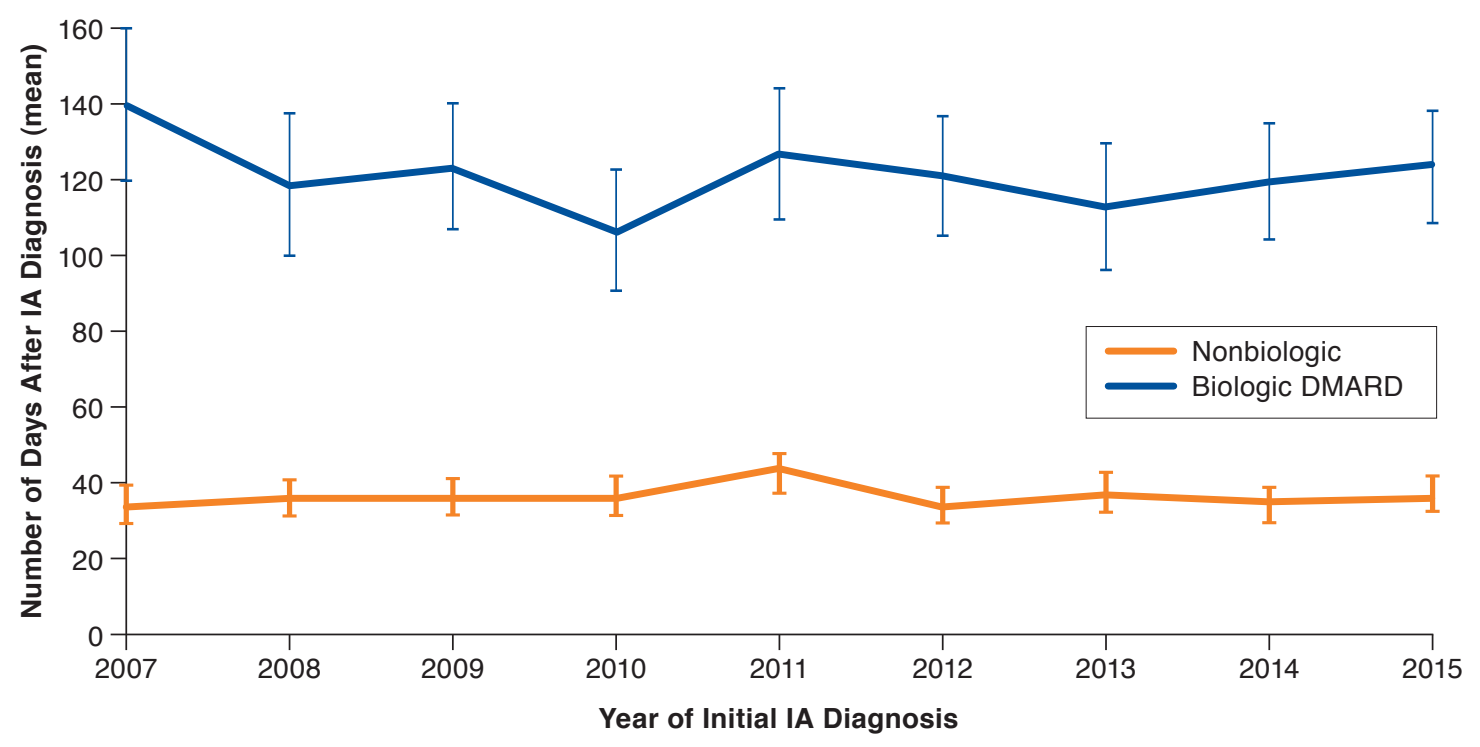

DMARD = disease-modifying antirheumatic drug; IA = inflammatory arthritis.

care from a rheumatologist. ${ }^{5,44-47}$ These associations between DMARD treatment and rheumatology care utilization suggest that prompt access to a rheumatology provider may be important for accessing DMARDs in a timely manner.

The percentage of patients with DMARD treatment during the 12 months after IA diagnosis increased over the years. These results demonstrated that VA providers gradually changed their prescribing patterns. These patterns may have been influenced by a growing acceptance, by providers and patients, of treat-to-target concepts that promote more aggressive therapies earlier in the course of disease. ${ }^{46-48}$ Furthermore, with expanding DMARD experience and safety literature, ${ }^{4,49-52}$ providers and patients may be increasingly comfortable with DMARD safety. Also, the lower rates of nontreatment over time may have been influenced by the selection of DMARD treatment as a HEDIS health care quality indicator in RA and recommendations for early treatment from professional organizations such as the ACR and EULAR. ${ }^{18,22,53,54}$

Among veterans with DMARD treatment within 12 months after diagnosis, treatment was initiated approximately 1-2 months after diagnosis, on average. There may be differences between patients with early treatment, delayed treatment, and no treatment in terms of demographics, disease severity, access to specialty care, comorbidities, and patient treatment preferences. Understanding the contextual factors associated with early, delayed, and nontreatment is necessary to identify modifiable patterns of treatment delays and nontreatment.

\section{Strengths}

A strength of this study is the large cohort with widespread geographic representation across the United States. Another advantage is that we were able to comprehensively review individual patient medical records to develop and test methods for accurately identifying patients with incident IA. Our rigorous cohort identification methods minimized erroneous inclusions of patients without IA or with prevalent IA.

\section{Limitations}

Study limitations included imperfect capture of incident IA cases, since some incident IA cases were inevitably excluded, and we were unable to eliminate all prevalent IA cases. Another weakness is that the exclusion of IA patients with DMARD treatment before the IA diagnosis date may have selected against PsA patients with severe psoriasis, since psoriasis typically occurs before PsA, ${ }^{55}$ and DMARD use is expected to be higher with severe psoriasis than mild psoriasis. Also, this study likely selected for AS patients with long diagnostic delays, since AS onset is typically before the age of 45 , and the mean age of the initial AS diagnosis in this population was 57.6 years. Thus, the PsA and AS patients in this study may not be closely representative of the general PsA and AS populations. The generalizability of the study findings to nonVA populations is also limited due to relatively unique aspects of the veteran population (i.e., primarily older males) and the VA health care system (i.e., funded and administered by the federal government). 


\section{Conclusions}

Many veterans were not treated with a DMARD in the first year after IA diagnosis, despite literature demonstrating that nontreatment and treatment delays are associated with qualityof-life impairments, disability, and irreversible joint damage. Additional research will expand our understanding of reasons for nontreatment and opportunities for improving care for individuals with IA.

\section{Authors}

JESSICA A. WALSH, MD; GOPI K. PENMETSA, MD; DANIEL O. CLEGG, MD; and GRANT W. CANNON, MD, Division of Rheumatology, Department of Internal Medicine, Salt Lake City Veterans Affairs and University of Utah Medical Centers. SHAOBO PEI, PhD; BRIAN C. SAUER, PhD; and VIKAS PATIL, MS, Division of Epidemiology, Department of Internal Medicine, Salt Lake City Veterans Affairs and University of Utah Medical Centers. JODI H. WALKER, PharmD, BCPS; JERRY CLEWELL, PharmD, MBA; and KEVIN M. DOUGLAS, MD, AbbVie, North Chicago, Illinois. AHMAD HALWANI, MD, Division of Hematology, Department of Internal Medicine, Salt Lake City Veterans Affairs and University of Utah Medical Centers.

AUTHOR CORRESPONDENCE: Jessica A. Walsh, MD, Division of Rheumatology, Department of Internal Medicine, Salt Lake City Veterans Affairs and University of Utah Medical Centers, 30 North 1900 East, Salt Lake City, UT 84132. Tel.: 801.581.7724;

E-mail: Jessica.walsh@hsc.utah.edu.

\section{DISCLOSURES}

AbbVie Pharmaceuticals and Marriott Daughters Foundation funded this study via investigator-initiated grants. Data analyses were completed by investigators independent of AbbVie and Marriott Daughters Foundation. Walker, Clewell, and Douglas are employed by, and stockholders in, Abbvie. Halwani reports grants from BMS, Kyowa Hakko Kirin, Seattle Genetics, Roche-Genentech, Miragen, Immunedesign, Takeda, Amgen, Pharmacyclics, and Abbvie. The other authors have nothing to disclose.

\section{REFERENCES}

1. Gladman DD, Antoni C, Mease P, Clegg DO, Nash P. Psoriatic arthritis: epidemiology, clinical features, course, and outcome. Ann Rheum Dis. 2005;64(Suppl 2):ii14-17.

2. Gonzalez CM, Carmona L, de Toro J, et al. Perceptions of patients with rheumatic diseases on the impact on daily life and satisfaction with their medications: RHEU-LIFE, a survey to patients treated with subcutaneous biological products. Patient Prefer Adherence. 2017;11:1243-52.

3. Ma VY, Chan L, Carruthers KJ. Incidence, prevalence, costs, and impact on disability of common conditions requiring rehabilitation in the United States: stroke, spinal cord injury, traumatic brain injury, multiple sclerosis, osteoarthritis, rheumatoid arthritis, limb loss, and back pain. Arch Phys Med Rehabil. 2014;95(5):986-95.el.

4. Ramonda R, Marchesoni A, Carletto A, et al. Patient-reported impact of spondyloarthritis on work disability and working life: the ATLANTIS survey. Arthritis Res Ther. 2016;18:78.
5. Walsh JA, Song X, Kim G, Park Y. Healthcare utilization and direct costs in patients with ankylosing spondylitis using a large U.S. administrative claims database. Rheumatol Ther. 2018;5(2):463-74.

6. van der Linden MP, le Cessie S, Raza K, et al. Long-term impact of delay in assessment of patients with early arthritis. Arthritis Rheum. 2010;62(12):3537-46.

7. Haroon M, Gallagher P, FitzGerald O. Diagnostic delay of more than 6 months contributes to poor radiographic and functional outcome in psoriatic arthritis. Ann Rheum Dis. 2015;74(6):1045-50.

8. Kimsey L, Weissman JS, Patel A, Drew A, Koehlmoos T, Sparks JA. Delay in initiation of DMARD or anti-inflammatory therapy in patients newly diagnosed with rheumatoid arthritis: an analysis of United States Military Health System TRICARE beneficiaries. Semin Arthritis Rheum. 2019;48(5):821-27.

9. Smolen JS, Landewe R, Bijlsma J, et al. EULAR recommendations for the management of rheumatoid arthritis with synthetic and biological disease-modifying antirheumatic drugs: 2016 update. Ann Rheum Dis. 2017;76(6):960-77.

10. Monti S, Montecucco C, Bugatti S, Caporali R. Rheumatoid arthritis treatment: the earlier the better to prevent joint damage. RMD Open 2015;1(Suppl 1):e000057.

11. Makinen H, Kautiainen H, Hannonen P, et al. Sustained remission and reduced radiographic progression with combination disease modifying antirheumatic drugs in early rheumatoid arthritis. J Rheumatol. 2007;34(2):316-21.

12. van Nies JA, Tsonaka R, Gaujoux-Viala C, Fautrel B, van der Helm-van Mil AH. Evaluating relationships between symptom duration and persistence of rheumatoid arthritis: does a window of opportunity exist? Results on the Leiden early arthritis clinic and ESPOIR cohorts. Ann Rheum Dis. 2015;74(5):806-12.

13. Gremese E, Salaffi F, Bosello SL, et al. Very early rheumatoid arthritis as a predictor of remission: a multicentre real life prospective study. Ann Rheum Dis. 2013;72(6):858-62.

14. Nell VP, Machold KP, Eberl G, Stamm TA, Uffmann M, Smolen JS. Benefit of very early referral and very early therapy with disease-modifying anti-rheumatic drugs in patients with early rheumatoid arthritis. Rheumatology (Oxford). 2004:43(7):906-14.

15. Lukas C, Combe B, Ravaud P, Sibilia J, Landew R, van der Heijde D. Favorable effect of very early disease-modifying antirheumatic drug treatment on radiographic progression in early inflammatory arthritis: data from the Etude et Suivi des polyarthrites indifferenciees recentes (study and followup of early undifferentiated polyarthritis). Arthritis Rheum. 2011;63(7):1804-11.

16. Coates LC, Moverley AR, McParland L, et al. Effect of tight control of inflammation in early psoriatic arthritis (TICOPA): a UK multicentre, openlabel, randomised controlled trial. Lancet. 2015;386(10012):2489-98.

17. Sieper J, Braun J. How important is early therapy in axial spondyloarthritis? Rheum Dis Clin North Am. 2012;38(3):635-42.

18. Singh JA, Saag KG, Bridges SL Jr, et al. 2015 American College of Rheumatology guideline for the treatment of rheumatoid arthritis. Arthritis Care Res (Hoboken). 2016;68(1):1-25.

19. Singh JA, Guyatt G, Ogdie A, et al. 2018 American College of Rheumatology/National Psoriasis Foundation guideline for the treatment of psoriatic arthritis. Arthritis Care Res (Hoboken). 2019;71(1):2-29.

20. Ward MM, Deodhar A, Akl EA, et al. American College of Rheumatology/Spondylitis Association of America/Spondyloarthritis Research and Treatment Network 2015 recommendations for the treatment of ankylosing spondylitis and nonradiographic axial spondyloarthritis. Arthritis Care Res (Hoboken). 2016;68(2):151-66.

21. Combe B, Landewe R, Daien CI, et al. 2016 update of the EULAR recommendations for the management of early arthritis. Ann Rheum Dis. 2017;76(6):948-59. 
22. Desai SP, Yazdany J. Quality measurement and improvement in rheumatology: rheumatoid arthritis as a case study. Arthritis Rheum. 2011;63(12):3649-60.

23. Schmajuk G, Solomon DH, Yazdany J. Patterns of disease-modifying antirheumatic drug use in rheumatoid arthritis patients after 2002: a systematic review. Arthritis Care Res (Hoboken). 2013;65(12):1927-35.

24. Fihn SD, Francis J, Clancy C, et al. Insights from advanced analytics at the Veteran Health Administration. Health Aff (Millwood). 2014;33(7):1203-11.

25. U.S. Department of Veterans Affairs. VA Informatics and Computing Infrastructure (VINCI). 2017. Available at: https://www.hsrd.research.va.gov/ for_researchers/vinci/. Accessed September 17, 2019.

26. Chapman BE, Lee S, Kang HP, Chapman WW. Document-level classification of CT pulmonary angiography reports based on an extension of the ConText algorithm. J Biomed Inform. 2011;44(5):728-37.

27. Harkema H, Dowling JN, Thornblade T, Chapman WW. ConText: an algorithm for determining negation, experiencer, and temporal status from clinical reports. J Biomed Inform. 2009;42(5):839-51.

28. Patil V, Rasmussen KM, Burningham Z, et al. Using natural language processing in radiology reports to identify the presence of metastatic disease in veterans with prostate cancer. Abstract 9. AVAHO Updates. August 1, 2017. Available at: https://www.mdedge.com/fedprac/avaho/article/146302/usingnatural-language-processing-radiology-reports-identify-presence. Accessed September 17, 2019

29. R Core Team. The R Project for Statistical Computing. 2018. Available at: https://www.r-project.org/. Accessed September 17, 2019.

30. Kern DM, Chang L, Sonawane K, et al. Treatment patterns of newly diagnosed rheumatoid arthritis patients from a commercially insured population. Rheumatol Ther. 2018;5(2):355-69.

31. Crane MM, Juneja M, Allen J, et al. Epidemiology and treatment of newonset and established rheumatoid arthritis in an insured U.S. population. Arthritis Care Res (Hoboken). 2015;67(12):1646-55.

32. Bonafede M, Fox KM, Watson C, Princic N, Gandra SR. Treatment patterns in the first year after initiating tumor necrosis factor blockers in realworld settings. Adv Ther. 2012;29(8):664-74.

33. Bonafede M, Johnson BH, Shah N, Harrison DJ, Tang D, Stolshek BS Disease-modifying antirheumatic drug initiation among patients newly diagnosed with rheumatoid arthritis. Am J Manag Care. 2018;24(8 Spec No.):SP279-SP285

34. Yazdany J, Tonner C, Schmajuk G. Use and spending for biologic disease-modifying antirheumatic drugs for rheumatoid arthritis among U.S. Medicare beneficiaries. Arthritis Care Res (Hoboken). 2015;67(9):1210-18.

35. Kirkham B, de Vlam K, Li W, et al. Early treatment of psoriatic arthritis is associated with improved patient-reported outcomes: findings from the etanercept PRESTA trial. Clin Exp Rheumatol. 2015;33(1):11-19.

36. Maksymowych WP, Morency N, Conner-Spady B, Lambert RG.

Suppression of inflammation and effects on new bone formation in ankylosing spondylitis: evidence for a window of opportunity in disease modification. Ann Rheum Dis. 2013;72(1):23-28.

37. McLaughlin M, Ostor A. Early treatment of psoriatic arthritis improves prognosis. Practitioner. 2014;258(1777):21-24, 3

38. Rudwaleit M, Listing J, Brandt J, Braun J, Sieper J. Prediction of a major clinical response (BASDAI 50) to tumour necrosis factor alpha blockers in ankylosing spondylitis. Ann Rheum Dis. 2004;63(6):665-70.
39. Dougados M, Behier JM, Jolchine I, et al. Efficacy of celecoxib, a cyclooxygenase 2-specific inhibitor, in the treatment of ankylosing spondylitis: a six-week controlled study with comparison against placebo and against a conventional nonsteroidal antiinflammatory drug. Arthritis Rheum. 2001:44(1):180-85.

40. Coates LC, Helliwell PS. Psoriatic arthritis: state of the art review. Clin Med (Lond). 2017;17(1):65-70

41. Sritheran D, Leung YY. Making the next steps in psoriatic arthritis management: current status and future directions. Ther Adv Musculoskelet Dis. 2015;7(5):173-86

42. Wilsdon TD, Whittle SL, Thynne TR, Mangoni AA. Methotrexate for psoriatic arthritis. Cochrane Database Syst Rev. 2019;1:CD012722.

43. Allard A, Antony A, Shaddick G, et al. Trajectory of radiographic change over a decade: the effect of transition from conventional synthetic diseasemodifying antirheumatic drugs to anti-tumour necrosis factor in patients with psoriatic arthritis. Rheumatology (Oxford). 2019;58(2):269-73.

44. Shipton D, Glazier RH, Guan J, Badley EM. Effects of use of specialty services on disease-modifying antirheumatic drug use in the treatment of rheumatoid arthritis in an insured elderly population. Med Care. 2004;42(9):907-13.

45. Ward MM, Leigh JP, Fries JF. Progression of functional disability in patients with rheumatoid arthritis. Associations with rheumatology subspecialty care. Arch Intern Med. 1993;153(19):2229-37.

46. Tucker LJ, Ye W, Coates LC. Novel concepts in psoriatic arthritis management: can we treat to target? Curr Rheumatol Rep. 2018;20(11):71.

47. Versteeg GA, Steunebrink LMM, Vonkeman HE, Ten Klooster PM, van der Bijl AE, van de Laar M. Long-term disease and patient-reported outcomes of a continuous treat-to-target approach in patients with early rheumatoid arthritis in daily clinical practice. Clin Rheumatol. 2018;37(5):1189-97.

48. Strand V, Wright GC, Bergman MJ, Tambiah J, Taylor PC. Patient expectations and perceptions of goal-setting strategies for disease management in rheumatoid arthritis. J Rheumatol. 2015:42(11):2046-54.

49. Holroyd CR, Seth R, Bukhari M, et al. The British Society for Rheumatology biologic DMARD safety guidelines in inflammatory arthritis. Rheumatology (Oxford). 2019;58(2):372.

50. Ramiro S, Sepriano A, Chatzidionysiou K, et al. Safety of synthetic and biological DMARDs: a systematic literature review informing the 2016 update of the EULAR recommendations for management of rheumatoid arthritis. Ann Rheum Dis. 2017;76(6):1101-36.

51. de La Forest Divonne M, Gottenberg JE, Salliot C. Safety of biologic DMARDs in RA patients in real life: a systematic literature review and metaanalyses of biologic registers. Joint, Bone, Spine. 2017;84(2):133-40.

52. Andersen KM, Kelly A, Lyddiatt A, et al. Patient perspectives on DMARD safety concerns in rheumatology trials: results from inflammatory arthritis patient focus groups and OMERACT attendees discussion. J Rheumatol. 2019;46(9):1168-72

53. Saag KG, Teng GG, Patkar NM, et al. American College of Rheumatology 2008 recommendations for the use of nonbiologic and biologic diseasemodifying antirheumatic drugs in rheumatoid arthritis. Arthritis Rheum. 2008;59(6):762-84

54. Singh JA, Furst DE, Bharat A, et al. 2012 update of the 2008 American College of Rheumatology recommendations for the use of disease-modifying antirheumatic drugs and biologic agents in the treatment of rheumatoid arthritis. Arthritis Care Res (Hoboken). 2012;64(5):625-39.

55. Eder L, Chandran V, Shen $\mathrm{H}$, et al. Incidence of arthritis in a prospective cohort of psoriasis patients. Arthritis Care Res (Hoboken). 2011;63(4):619-22. 
Treatment Patterns with Disease-Modifying Antirheumatic Drugs in U.S. Veterans with Newly Diagnosed Rheumatoid Arthritis, Psoriatic Arthritis, or Ankylosing Spondylitis

\section{APPENDIX A Study Design}

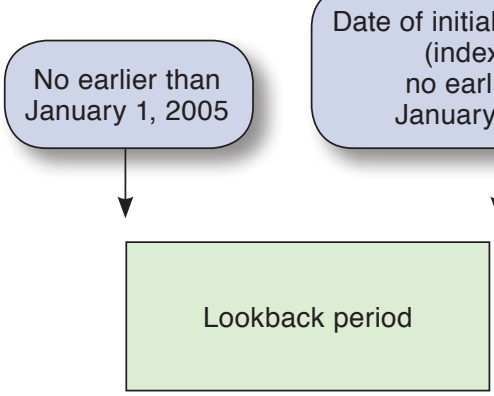

initial IA diagnosis

(index date)

no earlier than

anuary 1, 2007

No later than

December 31, 2015

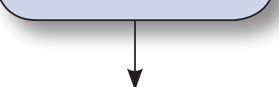

No later than

December 31, 2016

Study period (January 1, 2005-December 31, 2016)

IA = inflammatory arthritis.

\section{APPENDIX B Percentage of Veterans Who Accessed Rheumatology Care Before DMARD Initiation}

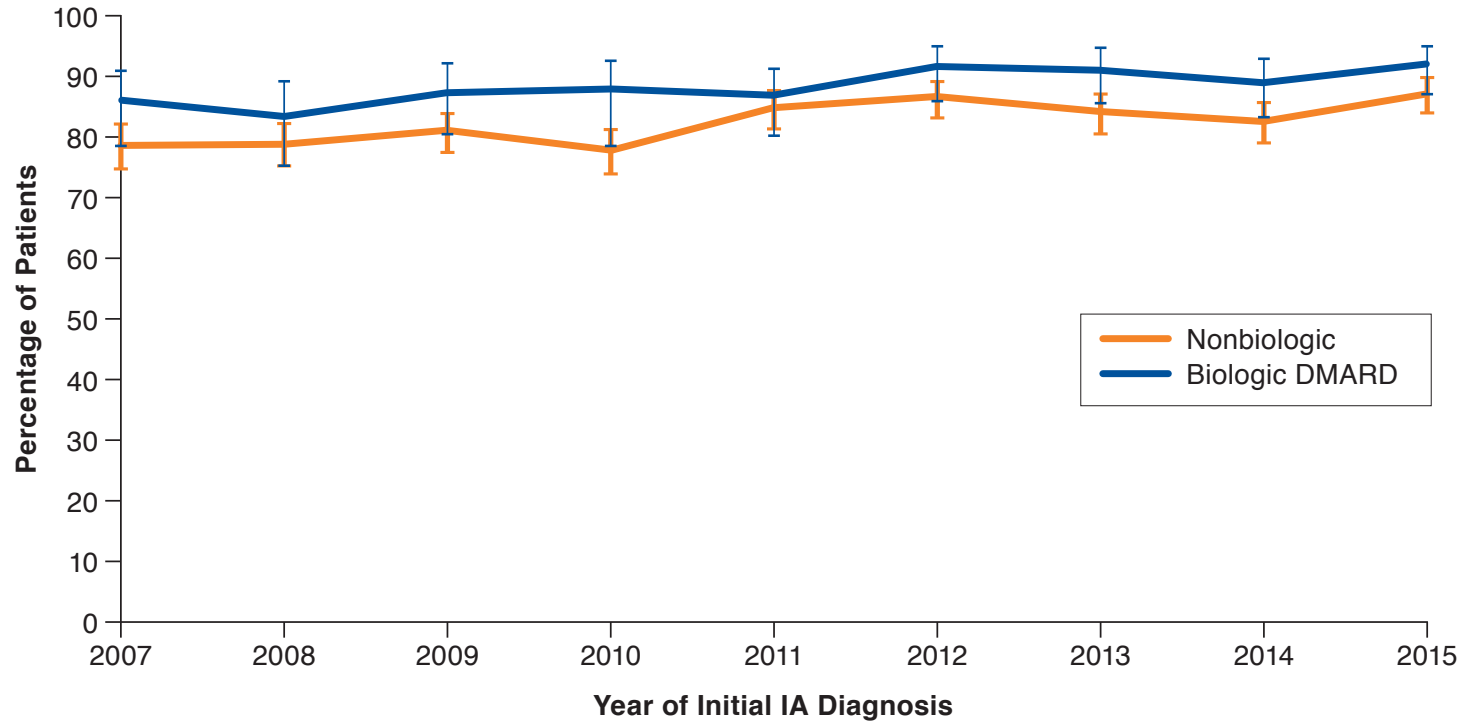

DMARD = disease-modifying antirheumatic drug; IA = inflammatory arthritis. 